\title{
Drei Aphorismen zur Entwickelungsphysiologie jüngster Stadien.
}

Von

\section{Hans Driesch.}

Mit 4 Figuren im Text.

Eingegangen am 12. Juli 1903.

\section{A. Über eine Modifikation der Versuche an isolirten Achterblastomeren von Echinus.}

Im Jahre $\left.1900^{1}\right)$ zeigte ich auf experimentellem Wege, dass das Ei der Seeigel Differenzen des Banes in Richtung seiner Achse besitzen müsse, und ein Jahr darauf theilte Boverr. ${ }^{2}$ ) unabhängig von meinen Untersuchungen gemachte Befunde mit, durch welche dieselben eine deskriptive Bestätigung willkommenster Art erhielten.

Ich hatte festgestellt, dass die Elemente des achtzelligen Stadiums sich verschieden verhalten, je nachdem sie der animalen oder der vegetativen (mikromerenliefernden, darmbildenden) Hälfte des Keimes entstammen, dass nämlich von den vegetativen Achtern ein weit höherer Procentsatz als von den animalen die Gastrulation vollzieht - um von anderen Differenzen hier abzusehen. Boveri stellte an Eiern von Strongylocentrotus sichtbare Bauverschiedenheiten des animalen und des vegetativen Eitheils fest: das Ei dieser Species ist in seinem animalen Theil durchsichtig weiß gefärbt, im vegetativen Theil besitzt es, an den Eiäquator angrenzend, einen breiten, orangefarbigen Ring, der fast bis zum vegetativen Pole reicht; die Polregion selbst ist wieder farblos.

1) Driesch, Die isolirten Blastomeren des Echinideneies. Arch. Entw.-Mech. 10. 1900. pag. 362.

2) Boverr, Über die Polarität des Seeigeleies. Verh. phys.-med. Ges. Würzburg. N. F. 34. 1901. pag. 145. 
Sind nun auch wohl begründete Bedenken dagegen geltend zu machen, wenn Boveri die drei Bestandtheile des StrongylocentrotusEies in unmittelbare Lokalisationsbeziehungen zur normalen Genese der drei sogenannten Keimblätter setzt ${ }^{1}$ ), so wird eine unbestimmte Zuordnungsbeziehung jener Regionen zu den Primitivorganen der Larve doch zuzugeben sein. Es verdient hier hervorgehoben zu werden, dass Boveri, wie angedeutet, für das durchaus "normale", ungestörte Geschehen zwar in jenen Regionen geradezu die Materialien für die Keimblätter sah, dass er aber; in richtiger Würdigung der vorliegenden Experimentaldata, sich solche Beziehungen nicht als absolut fix, sondern als mannigfach regulirbar vorstellte.

Ich hatte ursprünglich das so häufig. (in ca. $75 \%$ der Fälle) konstatirte Unvermögen animaler Achterzellen zur Gastrulation daraus zu erklären versucht, dass das Eiplasma mit fortschreitender Entwickelung immer starrer und daher weniger zum Ganzen regulirbar werde; nebenbei dachte ich auch an während des ersten Entwickelungsverlaufs stattfindende Stoffwanderungen, durch die wohl eine materielle Differenz der Blastomeren zu Stande käme.

Nach Boveri's Befunden schien es aber ganz plausibel, dass animale Achter desshalb zur Gastrulation meist nicht fähig seien, weil sie eben meist einen zu dieser Funktion nothwendigen »Stoff * nicht besäßen. BovenI hatte konstatirt, dass der orangefarbige Ring

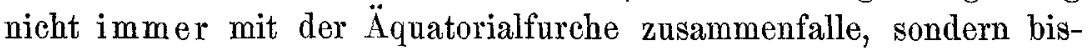
weilen über sie animalwärts hinausgreife: dass die Fälle der Gastrulation von animalen Achtern (ea. 25\%) mit Fällen dieses Übergreifens zusammenfielen, aus ihm zu erklären seien, erschien da ganz plausibel.

Ich schloss mich als $0^{2}$ ) im Wesentlichen BoverI's Deutung an, immerhin den Gedanken an ein "Starrerwerden « des Plasmas nicht ganz aus dem Bereich der Erklärungsmöglichkeiten verbannend. -

Im Verlaufe dieses Frühjahrs fiel mir nun eine Versuchsanordnung ein, durch die wohl eine gewisse Entscheidung zwisehen den für die verschiedene Entwickelung der Achterzellen des Echinidenkeimes aufgestellten Erklärungen getroffen werden konnte, und ich benutzte einen Aufenthalt in Neapel zur Durchführung der erforderlichen Experimente.

1) Hierzu: DriEsch, Nene Ergänzungen zur Entwickelungsphysiologie des Echinidenkeimes. Arch. Entw.-Mech. 14. 1902. pag. 500, und MoraAN, The Gastrulation of the Partial Embryos of Sphaerechinus. Ebenda. 16. 1903. pag. 117.

2) DrIeschr, Neue Ergänzungen etc. 
Im Jahre 1893 habe ich gezeigt ${ }^{1}$ ), dass Eier von Echinus in Seewasser, welches mit 20\% Flusswasser verdünnt ist, typische Abweichungen des Furchungsschemas zeigen, und zwar bestehen dieselben, so weit sie uns hier angehen, darin, dass das achtzellige Stadium sich nicht aus acht nahezu gleich großen Elementen zusammensetzt, dass vielmehr in einigen oder sogar in allen Quadranten eine durchaus inäquale Theilung eintritt, so dass im extremsten Falle der Keim aus vier "Makromeren " und vier »Mikromeren « besteht; daneben können Fälle mit etwa zwei Makro-, zwei Mikro- und vier "Meso"-meren vorkommen nnd Entsprechendes mehr (s. Fig. 1). Solche »vorzeitige "Mikromeren entsprechen örtlich dem auch sonst, aber nur ein Stadium später, mikromerenbildenden, also dem wahren vegetativen Pole des Eies.

Fig. 1.
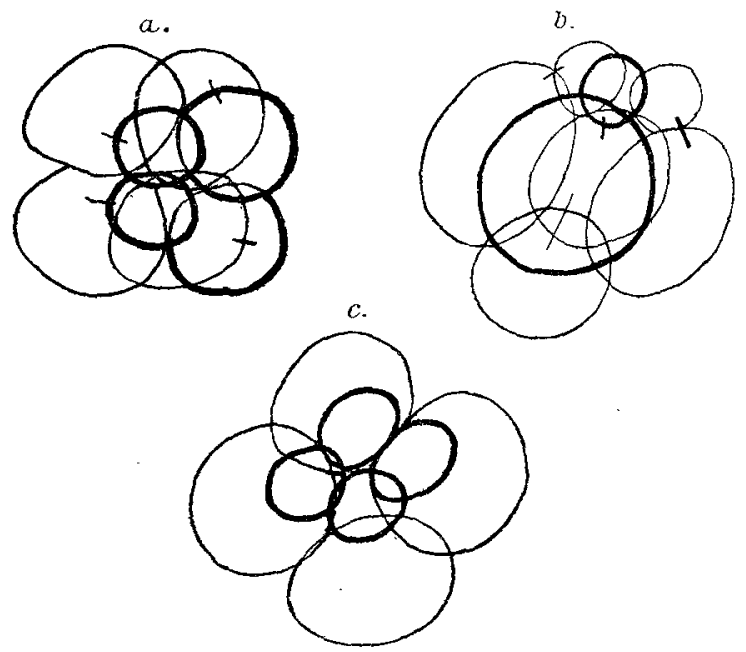

Echinus microtuberculatus. Achterstadien aus verdünntem Seewasser mit zwei $(a)$, drei $(b)$ nnd vier (c) vorzeitigen Mikromeren.

(Kopien aus Nr. VIII meiner sEntwickolungsmechanischen Studien.

Es ist nun klar, dass man in den "Makromeren « der soeben geschilderten abnormen Achterstadien Zellen vor sich hat, die im Sinne der Anschaungen Boveri's Bestandtheile von jedenfalls zweien seiner drei Eiregionen aufweisen. Liegt nun in dem Grade der Antheilnahme an den verschiedenen Eiregionen der Grund für die verschiedene Fähigkeit animaler Achterblastomeren zur Gastrulation, so

1) Driesch, Entwickelungsmechanische Studien. VIII. Mitth. Zool. Stat. Neapel. 11. 1893. pag. 226. 
war zu erwarten, dass "vorzeitige « Makromeren des Seeigelkeimes wohl stets oder doch beinahe stets gastrulationsvermöglich seien.

Es galt also, vorzeitige Makromeren des Achterstadiums von Echinus zu isoliren: nachdem die gleich nach der Befruchtung membranlos gemachten Eier bis zur Achtertheilung in mit 20\% Süßwasser verdünntem Seewasser gelegen hatten, wurden sie bei Beginn derselben in kalkfreies Wasser gebracht1); ein Anfenthalt von 15 Minuten genügte meist zum Zerfall in die Elemente. Nun wurden die entstandenen »Makromeren « (meist 2 oder 3, bisweilen alle 4, selten 1) sorgfältig herauspipettirt und wieder in normales Seewasser iibertragen.

Ich gehe zunäehst auf die Gesammtheit aller ausgefithrten Versuche ein:

178 isolirte *vorzeitige Makromeren" des achtzelligen Stadiums von Echinus microtuberculatus ergaben folgende Resultate:

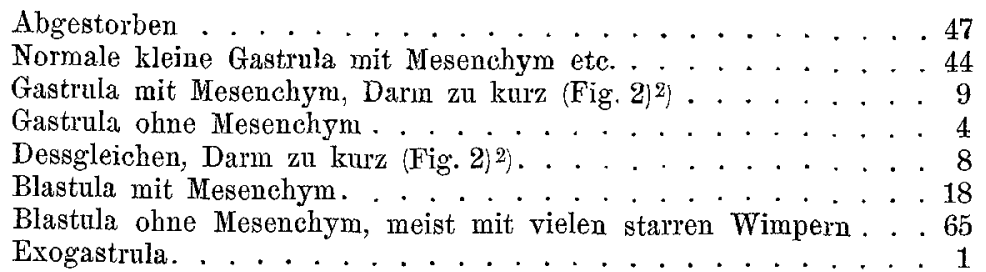

Fig. 2.

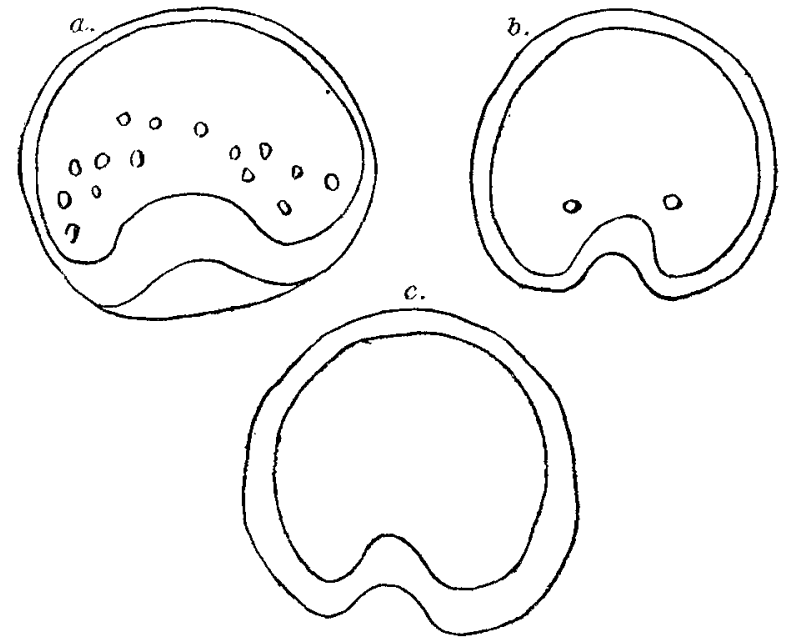

Echinus. Drei Gastrulac aus wvorzeitigen Makromerene des Achterstadiams nit zu kurzem Durm; $a$ besitzt normales Mesenchym, $c$ gar keines; $b$ hat an zwei Mesenchymzellen producirt. ZuISS $D^{*}$ ac. 4 .

1) Hierzu Herbst, Areh. Entw.-Mech. 9. 1900.

2) Abbilaungen der übrigen Kategorien finden sich in meinen $\gg$ Isolirten Blastomeren<. 
Seheiden wir die während der Furchung oder als junge Blastula abgestorbene Objekte (47) ans, so bleiben 131.

Von diesen kommen auf Bildungen, welche überhaupt, wenn auch nicht ganz normal, ganz oder theilweise gastrulirt haben, 66; durchaus nicht gastrulirt haben 65. Das macht für jede Kategorie mit sehr großer Annäherung $50 \%$ aus. Früher babe ich festgestellt, dass die normalen animalen Blastomeren des Achterstadiums bei Echinus nur in 23-25\% der Fälle gastruliren.

Eine Erhöhung des Procentsatzes ist also durch Verlegung der äquatorialen Furche nach dem vegetativen Pol zu - wie wir den von uns hervorgerufenen Process kurz nennen können - jedenfalls eingetreten. Eine andere Frage ist aber die, ob die Erhöhung derartig: ist, dass sie wirklich dazu zwingt, Boverr's Ansichten über den Grund des Vermögens oder Unvermögens der Achterzellen zu acceptiren.

Ich denke, dass solches nicht der Fall ist.

Dabei bemerke ich, dass ich bei Beginn meiner Versuche von der Richtigkeit der auf Boveri's deskriptive Befunde sich stützenden Schlussfolgerung ziemlich fest überzeugt war und eigentlich erwartete, $100 \%$ Gastrulae za erhalten.

In dem Umstand nun, dass zwar von 100 Procent Gastrulae in den modificirten Versuchen gar keine Rede war, während doch immerhin eine gewisse Erhöhung des Procentsatzes der Gastrulae zur Beobachtung gelangte, scheint mir ein hinreichender Grund zu liegen für den Schluss, dass Boveri's Auffassung zwar eine gewisse Berechtigung zukommt, dass aber meine alte Auffassung von einem Starrerwerden des Eiplasmas im Laufe der Entwickelung und von einem damit proportional abnehmenden Regulationsvermögen neben jener Ansicht durchaus zu Recht bestehen bleibt.

Wie sollte es sonst wohl möglich sein, dass sich die vorzeitigen Makromeren ein und desselben Keimes, deren jede doch gleichen Antheil an den regetativen Keimbestandtheilen besitzt, in Hinsicht ihrer Leistungen durchaus verschieden verhalten können, ob̉ne dass darum doch die weniger Leistenden »krank « sind oder etwa früh sterben? Hier muss es sich wohl um den geringeren oder größeren Grad des Stattfindens einer Regulation von irgend etwas Unbekanntem, einer Intimstruktur, zum Ganzen handeln, ein Process, über den Näheres anzugeben wir leider gänzlich außer Stande sind.

Doch sei zunächst auf das Phänomen der individuellen Verschiedenheit der vorzeitigen Makromeren als solches an der Hand weniger Beispiele näher eingegangen. 
Die nachstehende Tabelle giebt in einigen Proben eine Übersicht dariiber, wie verschieden sich. die aus einem Keim stammenden vorzeitigen Makromeren verhalten können. Die erste Vertikalkolumne giebt die Zahl der Makromeren an, die von jedem Keim im Achterstadium geliefert wurden, die Horizontalreihen enthalten die Leistungen der jeweils zusammengehörigen Keimtheile.

Tabelle.

\begin{tabular}{c|c|c|c|c|c|c|c}
\hline $\begin{array}{c}\text { Zahl der } \\
\text { vorzeitigen } \\
\text { Makro- } \\
\text { meren }\end{array}$ & $\begin{array}{c}\text { Davon } \\
\text { todt }\end{array}$ & $\begin{array}{c}\text { Normaie } \\
\text { Gastrula }\end{array}$ & $\begin{array}{c}\text { Normale } \\
\text { Gastrula, } \\
\text { aber Darm } \\
\text { za kurz }\end{array}$ & $\begin{array}{c}\text { Gastrula } \\
\text { ohne }\end{array}$ & $\begin{array}{c}\text { Gastrula } \\
\text { ohne Mesenchym } \\
\text { Darm zu } \\
\text { Kurz }\end{array}$ & $\begin{array}{c}\text { Blastula } \\
\text { mit } \\
\text { Mesenchym }\end{array}$ & $\begin{array}{c}\text { Blastula } \\
\text { ohne } \\
\text { Mesenshym }\end{array}$ \\
\hline 3 & - & 2 & 1 & - & - & - & - \\
3 & - & 1 & - & 1 & - & 1 & - \\
3 & - & - & 1 & - & - & 1 & 1 \\
3 & - & 2 & - & - & - & 1 & - \\
3 & - & 1 & - & - & 1 & - & 1 \\
3 & -- & 1 & - & - & 1 & - & 1 \\
4 & - & -1 & - & - & 2 & - & 2 \\
3 & 1 & 1 & - & 1 & - & - & - \\
3 & 1 & 1 & - & - & - & 1 & - \\
4 & - & 1 & 2 & - & - & 1 & -
\end{tabular}

Zur Kontrolle habe ich in einer Anzahl von Fällen auch jeweils die vier Partner des vierzelligen Stadiums beisammen isolirt. Es starb hier wohl manchmal der eine derselben oder es starben auch zwei, aber ein eigentlich verschiedenes Entwickelungsresultat, wo doch, wie es bei den Objekten obiger Tabelle der Fall war, alle Partner gesund und hell gewesen wären, kam fast nie vor. Kam in seltenen Fällen einer der Partner nicht über die Blastula hinaus, so war ihm Kränklichkeit in beinahe allen Fällen anzusehen.

Einmal nur kam in einer Kultur eine gesunde mesenchymlose Danerblastula (nebst zwei normalen Gastrulae und einer solchen mit zu kurzem Darm) zur Beobachtung. Dass es solche als Abkömmlinge vom Viererstadium geben $k \ddot{b} n n e$, wusste ich von früher ${ }^{1}$ ). Es wird wohl das Rechte treffen, wenn man ein vorzeitiges (»anachronistisches «)

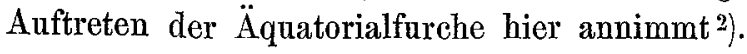

1) DRIEsch, Entwickelungsmeeh. Studien. IIIb. Zeitschr. wiss. Zool. 55. 1892. pag. 5 .

2) Dann wären also in Wahrheit zwei der Viertel »animal «, zwei »vegetativ«. Oder sollte in seltenen Fällen auch bei den $1 / 4$-Blastomeren die Intimregulation zum Ganzen ausbleiben können? 
Jedenfalls bildet ein verschiedenes Leistungsverhalten der vier Partner des Viererstadiums die sehr seltene Ausnahme; bei den Abkömmlingen eines Eies, falls sie vorzeitige Makromeren sind, bildet es die Regel.

Die eigentlich materialen Entwickelungsbedingungen sind hier offenbar für alle dieselben: da scheint mir in der That gar nichts Anderes tubrig zu bleiben als die Annahme, dass Verschiedenheiten in der Leistnng sich hier aus dem Inkrafttreten oder Unterbleiben einer Regulation zum Ganzen erkläre, einer Regulation, welche eben im achtzelligen Stadium dem vierzelligen gegenüber erschwert ist. Diese Regulation ist des Näheren durchans unbekannt, nur das können wir wohl sagen, dass ihre Auslösung in recht erheblichem Maße dem Zufall preisgegeben ist. Wer weil, von was für geringfügigen äußeren Bedingungen jene Auslösung hier abhängen mag? Man denke an die je nach der Lage so verschiedene Leistungsfähigkeit der Blastomeren des Froscheies 1).

Jedenfalls zeigen meine nenen Versuche, dass Antheilnahme an der vegetativen, bei Strongylocentrotus orange gefärbten, Partie des Eiplasmas noch nicht obne Weiteres eine Achterblastomere zur Gastrulation befähigt. -

Auf die Beziehungen der Formbildungsphänomene zum Eiplasmabau yerfen meine neuen Versuche nun auch noch von einer anderen Seite ein seltsames Licht:

Den vorzeitigen Makromeren fehlt jeweils ein Viertel des regetativen Eitheils, nämlich jenes Bezirks, dem BoverI für das "Normale" die Leistung der Mesenchymbildung zuschreibt.

Dass, wie die Übersicht auf pag. 44 zeigt, immerhin 12 Gastrulae ohne Mesenchym in meinen Versuchen zur Beobachtung gelangten, möchte man wohl versucht sein, damit in Beziehung zu setzen.

Aber wie steht es nun mit jenen 18 Fällen, in denen gesunde Blastulae mit Mesenchym, aber ohne Darm zur Beobachtung gelangten?

Mir scheint, dass diese Befunde doch jedes irgendwie nähere Inbeziehungsetzen von Eiregionen and Keimtheilen, wenigstens für unsere Versuche, hinfällig erscheinen lassen miissen.

Wir können die Gesammtheit unserer neneren und älteren Experimentalbefunde wohl nicht gut anders deuten als folgendermaßen:

1) Mordan, Anat. Anz. 10. 1895. 
Achterblastomeren der Echiniden vermögen eine vollständige Gastrula mit Mesenchym (und Skeletansatz) 'zu produciren, falls sie einen gewissen Antheil des regetativen Eiplasmas enthalten, und zwar steigt die Wahrscheinlichkeit zu einer vollständigen Leistung mit dem Wachsen jenes Antheils am vegetativen Eiplasma. Voraussetzung für eine vollständige Leistung ist aber das Statthaben einer in ihren näheren Umständen unbekannten, wahrscheinlich in ihrem Eintreten dem "Zufall " in hohem Maße ausgesetzten Intimregulation zum Ganzen. Unterbleibt diese oder geschieht sie unvollständig, so wird entweder nur eine Blastula ohne jede weitere Organisation geliefert, oder eine Larve, die in irgend einem Organsystem, sei es der Darm oder das Mesenchym, Unvollständigkeiten aufweist. -

\section{B. Von der Entwickelung vor der Befruchtung verschmolzener Eier.}

Schon vor einigen Jahren theilte mir mein Freund HERBsT mit, dass er an Eiern von Echinus microtuberculatus am Ende der Reifezeit (im Mai) häufig Verschmelzungsprocesse beobachtet habe, und zwar seien diese dann aufgetreten, wenn zu jener Zeit unbefruchtete Eier etwa 24 Stunden lang in größeren Mengen bei einander gelegen hatten. Versuche, die nachträglich befruchteten Versehmelzungsprodukte zur Entwickelung zu bringen, schlugen Herbst fehl.

Da ich dieses Jahr des Amphioxus wegen doch den ganzen Mai in Neapel zuzubringen hatte, nahm ich die Untersuchung der Verschmelzungseier des Echinus auf ibr Entwickelungsvermögen hin vor. Es war ja klar, dass sich hier wichtige Beziehungen zu jenen Phänomenen ergeben kounten, die ich 1900 an Sphaerechinus-Keimen, welche wahrscheinlich als späte Furchungsstadien oder als junge Blastulae verschmolzen waren, beobachtet hatte ${ }^{1}$ ).

Fast bei jedem Versuch erhielt ich an Eiern von Echinus in der zweiten Hälfte des Mai wieder jene von HeRBsT zuerst beobachteten Objekte, wenn ich dieselben 24-30 Stunden lang, in nicht gar zu großer Menge, aber recht dicht gedrängt, bei einander liegen lieb.

An der Zahl der, mit einer Ansnahme, isolirt gebliebenen Kerne ließ sich, von den Größenverhältnissen abgesehen, unschwer der Ursprung der Verschmelzungsobjekte erkennen: ich habe 50 aus zwei,

1) Driesch, Studien über das Regulationsvermögen. IV. Arch. Entw.-Mech. 0. 1900. pa g. 411 . 
7 ans drei, 5 aus vier und 1 aus sechs Eiern zusammengeschmolzene Produkte isolirt und anf die Entwickelungsfähigkeit hin geprüft.

Von allen diesen 63 objekten furchte sich (unregelmäßig und nicht näher deutbar) nach vorgenommener Befruchtung 1) etwa die Hälfte; etwa ein Viertel ergab sogar eine kränkliche trübe Blastula. Aber alle Objekte bis auf drei sind dann während der Furchung oder als Blastula abgestorben, wofern überhaupt Entwickelung bei ibnen eingeleitet gewesen war.

Die drei Objekte, welche weitere Entwickelung zeigten, repräsentirten vollkommen typisch zwei der Kategorien, die ich 1900 als Ergebnisse verschmolzener Jungblastulae erhalten hatte: Eines lieferte eine Gastrula mit zwei dicht neben einander verlaufenden Därmen und weiterhin einen der Form und dem Skelet nach einheitlichen Pluteus, dessen (gegliederte) Därme gerade in Verschmelzung begriffen waren, als das Objekt abstarb; Fig. 7 meiner Arbeit von 1900 kann etwa als Bild dieses Objektes geiten. Die zwei anderen entwickelungsfähigen Verschmelzungskeime gastrulirten doppelt an verschiedenen Orten des gemeinsamen Blastoderms, einer derselben starb als Doppelgastrula, der andere entsprach etwa der Fig. 2 meiner citirten Arbeit.

Eier, welche vor der Befruchtung zu einem nenen Großganzen verschmolzen sind, können sich also nach Befruchtung entwickeln und folgen dann den für anderweitige Verschmelzungsprodukte geltenden Regeln. Thatsächlich findet aber Entwickelung nur sehr selten statt.

Dass alle diese Befunde großes cytologisches Interesse bieten, ist ohne Weiteres klar.

Einmal wies ein Zweierverschmelzungsprodukt nur einen großen Kern auf; das war vielleicht ein Analogon zu Zur Strassen's Rieseneiern 2). Leider leistete es nicht einmal die Furchung. -

Für die Frage nach dem Aggregatzustand des Protoplasmas (BǘSSCHLr, RHUMBLER) ist die Verschmelzung zweier oder mehrerer kugeliger Eier zu einem neuen vollk ommen kugeligen Gebilde natïrlich auch bedeutungsvoll; frèi suspendirte Flüssigkeitstropfen verhalten sich bekanntlich ebenso. -

1) Eine Membran wird nach so langem Liegen durch die Befruchtung meist nicht einmal bei normalen Einzeleiern hervorgerufen.

2) Zur Strassen, Arch. Entw.-Mech. 7. 1898. 


\section{C. Über die Größe und Zahl der Somiten bei Kleinlarven des Amphioxus.}

a. Vorbemerkungen.

Die wachstehend beschriebenen Untersuchungen habe ich in den letzten zwei Dritteln des Mai 1903 am Neapler Amphioxns ansgefiilurt; mit gewohnter Zuvorkommenheit ward mir fast au jedem Nachmittag der kleinere der beiden Dampfer der Zoologischen Station zur Verfiigung gestellt.

Die jungen Keime des Neapler Amphioxus verhalten sich wesentlich anders als diejenigen des Amphioxus rom Faro bei Messina. Für den sicilianischen Amphioxus giebt WILson ${ }^{1}$ ) an, dass man durch sehr geringfügiges Schütteln schon seine Blastomeren trennen kömne, und ich vermochte 1895 diese Angaben zu bestätigen. In Neapel erreichte ich durch Schütteln gar nichts als höchstens den Tod oder sogar das Wiederzusammengehen der Furchungszellen. HuRbs'r's kalkfreies Wasser leitete hier zum Ziele, aber auch nur, wenn man der Lockerung des Zellenverbandes noch etwas durch wiederholtes Pipettiren nachhalf.

Die Laichrerhältnisse des Neapler Amphioxus sind ebenso unregelmäßig. wie die des sicilianischen; trotz zwölf vorgenommener Ausfahrten erhielt ich nur dreimal Eimaterial, am 12.; 19. und 27. Mai. -

Über die Größenverhältnisse von aus isolirten Blastomeren gezogenen Kleinkeimen habe ich im Jahre 1900 die Angabe gemacht 2), dass ihre Flächen stets in Proportionalität zum »Keimwerth «, d. h. zu dem vom Versuchsobjekt repräsentirten Gesammtkeimbruchtheil, stehen; die Totalvolumina sind also stets zu klein, sie betragen z. B. bei $1 / 4$-Keimen nur $1 / 8$. Anlässlich eines Berichtes über neuere entwickelungsphysiologische Forschungen ${ }^{3}$ ) habe ich bereits darauf hingewiesen, dass man aus WILsox's Figuren zu seiner Amphioxus-Arbeit denselben Schluss ziehen kann, den ich aus dem Studium der Echinidementwickelung gewann. Ich füge hier bei, dass auch gewisse Angaben Mongan's 4), die ich im Jahre 1897 diskutirt habe ${ }^{5}$, denselben Schluss gestatten: Mongan giebt an, dass die $1 / 2$-Larven des Amphioxus zwei Drittel, die $1 / 4$-Larven die Hälfte der normalen

1) WiLson, Journ. Morph. 8. 1893, pag. 587.

2) Drresch, Arch. Entw.Mech. 10. 1900. pag. $393 \mathrm{f}$.

3) DrIEsch, Ergebnisse d. Anat. u. Entw. für 1901. pag. 930.

4) Murgan, Arch. Entw.Mech. 3. 1896. pag. 269.

5) Driesch, Arch. Entw.-Mech. 6. 1898. pag. $220 \mathrm{f}$. 
Länge der Larven entsprechenden Stadiums besäßen, und von diesen Angaben stimmt die erste naheza, die zweite exakt mit dem Verhalten, welches durch meinen allgemeinen Proportionalitätssatz gefordert wird.

Ein Blick auf die dieser Notiz beigegebenen Figuren wird zeigen, dass auch meine eigenen neuen Beobachtungen für die Richtigkeit des von mir vertretenen Satzes von der Proportionalität der Keimesflächen zum Keimwerth sprechen: in Fig. 3 ist der Kopf einer normalen und einer 1/2-Blastomerenlarve, zwei Tage nach der Befruchtung, von der Seite gesehen dargestellt; Fig. 4, welche sogleich näher zur Diskussion kommen wird, zeigt 22 Stunden alte, ganze, halbe

Fig. 3.

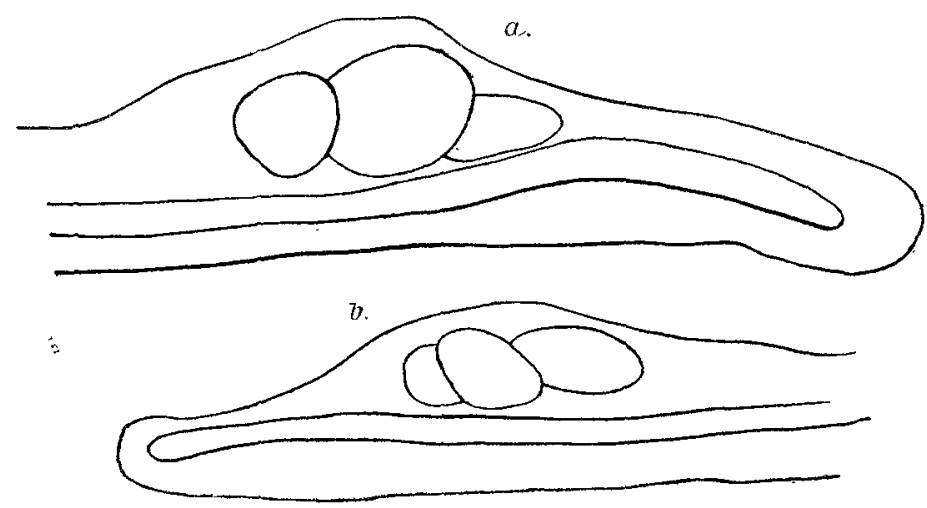

Amphioxus. Köpfe ron zwei Tage alten Larven. $a$ normal, $b$ aus einer 1/2-Blastomere.

und viertel Larven: an beiden Figurenserien verhalten sich die linearen Dimensionen ganz oder doch sehr stark angenähert so, wie sie sich nach meinem Satze verhalten müssen. -

Über die Entwickelungsgeschwindigkeit der Kleinlarven füge ich endlich noch die kurze Notiz bei, dass sie, ebenso wie bei Echiniden, mit abnehmender Keimesgröße um gewisse Beträge verzögert wird. Nach 16 Stunden waren beispielsweise die Normallarven im Stadium der gestreckten Gastrula mit Medullarrobr; die 1/2-Larven besaßen meist noch kein Medullarrohr, aber einen geschlossenen, an einem Ende gelegenen Blastoporus und bei den $1 / 4^{-}$ Larven war der Blastoporus etwa noch zur Hälfte offen; die Figuren 868, $867 C, 867 B$ bei KorschelT-Heider (pag. 1431f.) können etwa als Repräsentanten der' drei geschilderten Stadien dienen. Nach 22 Stunden waren die drei Kategorien der Keime einander organi- 
satorisch ähnlicher und unterschieden sich nur durch die Zahl der abgeschniirten Somiten (Fig. 4), welche mit abnehmendem Keimwerth abnahm, nicht sehr nennenswerth von einander.

b. Größe und Zahl der Somiten.

Als ich gefunden hatte 1 , dass längsgespaltene Stämme von Tubularia Tentakel in normaler Größe, aber, der verminderten Cönosarkoberfläche entsprechend, in verminderter Zahl bilden, dass also die Tentakel hier gleichsam eine höhere Einheit (den Zellen entsprechend, deren Größe und Form ja auch stets ${ }^{2}$ ) gewahrt bleibt)

Fig. 4.

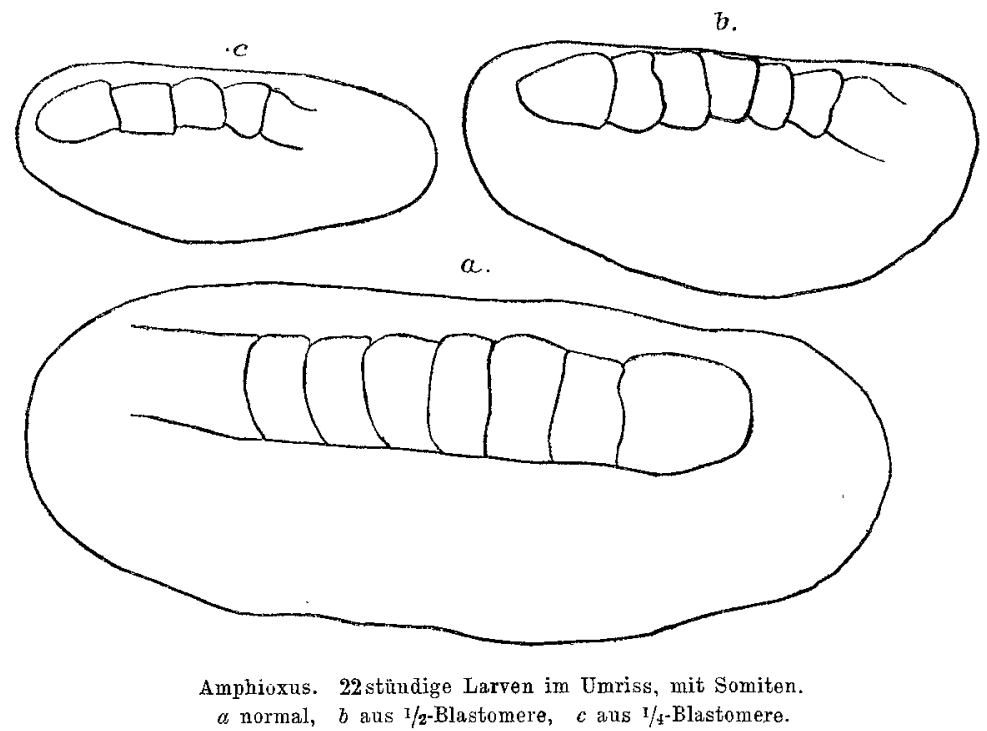

darstellen, vermuthete ich, dass die Metameren meristisch gebauter Thiere sich in Kleinkeimen vielleicht auch entsprechend verhalten möchten, dass auch sie vielleicht »höhere Einheiten " darstellen möchten, deren Größe eine unabänderliche sei.

Um die Richtigkeit meiner Vermuthung zu prüfen, plante ich schon seit Längerem Versuche an Amphioxus.

Inzwischen lernte ich eine fragmentarische Notiz von HERLITZKA ${ }^{3}$ ) kennen, in welcher für den Tritonkeim eine Abnahme der Zahl der

1) Driesch, Arch. Entw.-Mech. 11. 1901. pag. 199 f.

2) Wenigstens bei gleichen Kernverhältnissen.

3) Herlitzka, Arch. Ital. Biol. 35. 1901. pag. 132. 
Somiten (ohne Angabe über ihre Größe) mit abnehmendem Keimwerth kurz namhaft gemacht wird. -

Meine Beobachtungen an Amphioxus-Keimen zeigten mir gleich das erste Mal, dass hier sicherlich die einzelnen Somiten ihrer ab. soluten Größe nach keine »höheren Einheiten s darstellen.

In den Figuren 4 sind 22 Stunden alte Amphioxus-Larven verschiedenwerthigen Ursprungs dargestellt, welche ïber das Gesagte nicht den geringsten Zweifel lassen: die linearen Abmessungen der Somiten der $1 / 4$-Larven betragen ziemlich genau die Hälfte der normalen Längen, die Abmessungen der $1 / 2$-Larven betragen etwa $2 / 3$ derselben. Die Somiten sind also in ganz demselben Maßverhältnisse verkleinert, wie es alle Organe der Kleinlarven des Amphioxus sind.

Die Zahl der Somiten ist allerdings, wie schon am Ende des vorigen Abschnittes erwähnt, mit abnehmendem Keimwerth nach gleich langer Entwickelungszeit etwas verkleinert. Es wäre also vielleicht möglich, dass Kleinkeime nie zu der absoluten Zahl der Somite, welche Normallarven aufweisen, gelangen. Wahrscheinlicher erscheint mir jedoch, dass diese absolute Zahl auch von Kleinkeimen, nur etwas später, erreicht wird. Durch Beobachtung habe ich diese Frage minderer Bedeutung nicht entschieden.

Heidelberg, 7. VII. 1903. 